\title{
Renormdynamics and production of fractals, fluctons, solitons, strings, unparticles, ...
}

\author{
Nugzar Makhaldiani ${ }^{1, \star}$ \\ ${ }^{1}$ Joint Institute for Nuclear Research, Dubna, Moscow Region, Russia
}

\begin{abstract}
Concise introduction in renormdynamics with solvable models of (non)perturbative QCD and exotic states of fractals, fluctons, solitons, strings, unparticles...
\end{abstract}

We say that we find New Physics (NP) when either we find a phenomenon which is forbidden by SM in principle - this is the qualitative level of NP - or we find a significant deviation between precision calculations in SM of an observable quantity and a corresponding experimental value.

In QFT existence of a given theory means, that we can control its behavior at some scales (short or large distances) by renormalization theory [3]. If the theory exists, then we want to solve it, which means to determine what happens on other (large or short) scales. This is the problem (and content) of Renormdynamics. The result of the Renormdynamics, the solution of its discrete or continuous motion equations, is the effective QFT on a given scale (different from the initial one).

We will call Renormdynamics Functions (RDF) functions $g_{n}=f_{n}(t)$ which are solutions of the RD motion equations

$$
\dot{g}_{n}=\beta_{n}(g), 1 \leq n \leq N .
$$

In the simplest case of one coupling constant the function $g=f(t)$ is constant, $g=g_{c}$ when $\beta\left(g_{c}\right)=0$, or is invertible (monotone). Indeed,

$$
\dot{g}=f^{\prime}(t)=f^{\prime}\left(f^{-1}(g)\right)=\beta(g) .
$$

Each monotone interval ends by UV and IR fixed points and describes corresponding phase of the system. Note that the simplest case of the classical dynamics, the Hamiltonian system with one degree of freedom, is already two-dimensional, so we have no analog of one charge renormdynamics.

In the string theory, the connection between conformal invariance of the effective theory on the parametric world sheet and the motion equations of the fields on the embedding space is well known [8]. A more recent topic in this direction is AdS/CFT Duality [10]. In this approach for QCD coupling constant the following expression was obtained [2]

$$
\alpha_{A d S}\left(Q^{2}\right)=\alpha(0) e^{-Q^{2} / 4 k^{2}} .
$$

A corresponding $\beta$-function is

$$
\beta\left(\alpha_{A d S}\right)=\frac{d \alpha_{A d S}}{d \ln Q^{2}}=-\frac{Q^{2}}{4 k^{2}} \alpha_{A d S}\left(Q^{2}\right)=\alpha_{A d S}\left(Q^{2}\right) \ln \frac{\alpha_{A d S}\left(Q^{2}\right)}{\alpha(0)} \leq 0
$$

\footnotetext{
^e-mail: mnv@jinr.ru
} 
So, this renormdynamics of QCD interpolates between the IR fixed point $\alpha(0)$, which we take as $\alpha(0)=2$, and the UV fixed point $\alpha(\infty)=0$. For the QCD running coupling considered in [4]

$$
\alpha\left(q^{2}\right)=\frac{4 \pi}{9 \ln \left(\frac{q^{2}+m_{g}^{2}}{\Lambda^{2}}\right)},
$$

where $m_{g}=0.88 \mathrm{GeV}, \Lambda=0.28 \mathrm{GeV}$, the $\beta$-function of renormdynamics is

$$
\beta(\alpha)=-\frac{\alpha^{2}}{k}\left(1-c \exp \left(-\frac{k}{\alpha}\right)\right), k=\frac{4 \pi}{9}=1.40, c=\frac{m_{g}^{2}}{\Lambda^{2}}=(3.143)^{2}=9.88,
$$

for a nontrivial (IR) fixed point we have $\alpha_{I R}=k / \ln c=0.61$. For $\alpha(m)=2$, at valence quark scale $m$ we predict the gluon (or valence quark) mass as

$$
m_{g}=\Lambda e^{\frac{k}{2 \alpha(m)}}=1.42 \Lambda=m_{N} / 3, \Lambda=220 \mathrm{MeV} .
$$

It is nice to have a nonperturbative $\beta$-function like (6), but it is more important to see which kind of nonperturbative corrections we need to have a phenomenological coupling constant dynamics. It was noted [18] that in valence quark parameterization $\alpha_{s}(m)=2$, at a valence quark scale $m$.

In the 1870's G. J. Stoney [17], the physicist who coined the term "electron" and measured the value of elementary charge $e$, introduced as universal units of Nature for $L, T, M$ :

$$
l_{S}=\frac{e}{c^{2}} \sqrt{G}, t_{S}=\frac{e}{c^{3}} \sqrt{G}, m_{S}=\frac{e}{\sqrt{G}}
$$

M. Planck introduced [15] as universal units of Nature for L, T, M:

$$
m_{P}=\sqrt{\frac{h c}{G}}=\frac{m_{S}}{\sqrt{\alpha}}, l_{P}=\frac{h}{c m_{P}}=\frac{l_{S}}{\sqrt{\alpha}}=11.7 l_{S}, t_{P}=\frac{l_{P}}{c}=\frac{t_{S}}{\sqrt{\alpha}}
$$

Stoney's fundamental constants are more fundamental just because they are less than Planck's constants :) Due to the value of $\alpha^{-1}=137$, we can consider relativity theory and quantum mechanics as deformations of the classical mechanics when deformation parameter $c=137$ (in units $e=1, \hbar=1$ ) and $\hbar=137$ (in units $e=1, c=1$ ), correspondingly. These deformations have an analytic sense of p-adic convergent series. The number 137 has a very interesting geometric sense, $137=11^{2}+4^{2}$. So, $\sqrt{137}$ is the hypotenuse length of a triangle with other sides of lengths 11 and 4 . The Babylonians used a base 60 number system which is still used for measuring time - 60 seconds in a minute, 60 minutes in an hour - and for measuring angle - 360 degrees in a full turn. The base 60 number system has its origin in the ratio of the Sumerian mina (m) and Akkadian shekel (s), $m / s \simeq 60=3 \cdot 4 \cdot 5$. We also can consider base 137 system for fundamental theories. For the nuclear physics strong coupling phenomena description we may take as a base $p=13$. For the hadronic physics, valence scale QCD, and graphen strong coupling phenomena description we may take as a base $p=2$. For the weak coupling physics SM $m_{Z}$ scale and MSSM unification scale phenomena description we may take as a base $p=29$. There are different opinions about the number of fundamental constants [5]. According to Okun, there are three fundamental dimensionless constants in Nature: Planck's constant, $\hbar$; the velocity of light, $c$; and Newton's constant, $G$. According to Veneziano, there are only two: the string length $L_{s}$ and $c$. According to Duff, there are no fundamental constants at all.

Usually $L_{s}=l_{p}$, so, the fundamental area is $L_{s}^{2}=137 l_{s}^{2}$. The value $s_{s}=l_{s}^{2}-$ Stoney area, is more like on a fundamental area :) In mathematics we have two kind of structures, discrete and continuous. 
If a physical quantity has discrete values, it might have no dimension. If the values are continuous the quantity might have a dimension, a unit of measure. These structures may depend on scale, e.g. on macroscopic scale condensed state of matter (and time) is well described as continuous medium, so we use dimensional units of length (and time). On the scale of atoms, the matter has a discrete structure, so we may count lattice sites and may not use a unit of length. If at small (e.g. at Plank) scale space (and/or time) is discrete, then we do not need a unit of length (time) for measuring, there is a fundamental length and we can just count.

Let us consider the following discrete dynamics:

$$
S_{n+1}+S_{n-1}=\Phi\left(S_{n}\right)
$$

which is obviously a (discrete) time (n) invertible in this implicit form. In the explicit form

$$
S_{n+1}=F\left(S_{n}, S_{n-1}\right)=\Phi\left(S_{n}\right)-S_{n-1}
$$

it is not obviously time invertible. If we take two step time lattice-make simplest discrete RD step and from one component-scalar $\mathrm{S}(\mathrm{n})$ construct two component-spinor $\Psi(n)$, we obtain explicit time invertible dynamics

$$
\Psi_{n+1}=\Omega\left(\Psi_{n}\right), \Psi_{n+1}=\left(\begin{array}{c}
S_{n+2} \\
S_{n+1}
\end{array}\right), \Psi_{n}=\left(\begin{array}{c}
S_{n} \\
S_{n-1}
\end{array}\right) .
$$

This dynamical mechanism of origin spin which connects time inversion symmetry and spin was invented when the theory of quanputers [12] was constructed. This mechanism indicates that with time inversion symmetry we can have only composed scalar fields. With the discovery of the Higgs particle with mass $125 \mathrm{GeV}$, a nice number $m_{W} / m_{H} \simeq 80 / 120=2 / 3$ appears, which, at least for me, indicates for composed nature of $W$ and $H$, with the same mass of about $40 \mathrm{GeV}$ two and three valence constituents respectively. The fermion constituents $\psi_{n}^{a}$ of $W$ and scalar constituents $\varphi_{n}^{a}$ of $H$ compose scalar super multiplet $\left(\varphi_{n}^{a}, \psi_{n}^{a}\right)$ with a flavor index $n$ and color index $a$. Another notation is (h, sh)-(He, She:). The $40 \mathrm{GeV}$ constituents may be a good candidates for dark matter particles. Coupling constant unification at $\alpha_{u}^{-1}=29.0$ and scale $10^{16} \mathrm{GeV}$ in MSSM [13] has a relict on the SM scale: $\alpha_{2}^{-1}(m)=29.0$ at $m=41 \mathrm{GeV}$. If we extrapolate the SM value of $\alpha^{-1}\left(m_{Z}\right)$ to electron mass scale, we find $\alpha^{-1}\left(m_{e}\right)=137.0$ Recent discovery of the second Higgs particle with mass $M_{H}=750 \mathrm{GeV}$ indicates an interesting structure. It is curious that $M_{H} / m_{h}=750 / 125=6$ !

In the Standard Model of Particle Physics (SM), the values of the coupling constants and masses of particles depend on the scale according to the renormdynamic motion equations. One charge $a$, one mass $m$ RD equations are

$$
\dot{\alpha}=\beta(\alpha), \dot{m}=\gamma(\alpha) m .
$$

For the electron and nucleon masses, electrodynamic and pion-nucleon fine structure constants we have an empirical relation: $m_{e} / \alpha \simeq m_{N} / \alpha_{\pi N}$. We take the relation $m / \alpha=$ const as an integral of renormdynamics motion equations for $m$ and $\alpha$, find exact form of the $\beta$ function in the minimal mass parameterization

$$
\begin{aligned}
& \gamma(\alpha)=\gamma_{1} \alpha+\gamma_{2} \alpha^{2}+\ldots=\gamma_{1} A \\
& A=f^{-1}(\alpha)=\alpha+\gamma_{2} / \gamma_{1} \alpha^{2}+\gamma_{3} / \gamma_{1} \alpha^{3}+\ldots \\
& \alpha=f(A)=A+f_{2} A^{2}+f_{3} A^{3}+\ldots
\end{aligned}
$$

From the integral of motion, in the minimal mass parameterization: $\gamma(\alpha)=\gamma_{1} \alpha$, we obtain

$$
(\ln \alpha)^{\cdot}=(\ln m)^{\cdot} \Rightarrow \beta(\alpha) / \alpha=\gamma(\alpha)=\gamma_{1} \alpha \Rightarrow \beta(\alpha)=\beta_{2} \alpha^{2}, \beta_{2}=\gamma_{1}
$$


so, we have the following algebraic-diofant equations for the flavor and color content of the theory

$$
\beta_{2}=\gamma_{1}, \beta_{n}=0, n \geq 3,
$$

and prediction for the dimension of space-time: $D=4$. Solutions of the motion equations are

$$
\alpha(t)=\frac{\alpha_{0}}{1-\alpha_{0} \beta_{2} t}, m(t)=m_{0}\left|\alpha_{0}^{-1}-\beta_{2} t\right|^{-\gamma_{1} / \beta_{2}}=\frac{m_{0}}{\alpha_{0}} \alpha(t) .
$$

In the multidimensional renormdynamics, when we have several $(N)$ coupling constants and masses, we assume that there is maximal number $(N-1)$ of integrals of motion $H_{n}$. If the number of integrals is $N$, we do not have dynamics, we have only statics - finite field theory, $\alpha_{n}=$ const, $n=1, \ldots, N$. The idea of reduction to the one dimensional renormdynamics is simple:

$$
\begin{aligned}
& \frac{d \alpha_{n}}{d t}=\beta_{n}\left(\alpha_{1}, \ldots, \alpha_{(N-1)}, \alpha_{N}\right) \Rightarrow \frac{d \alpha_{n}}{d \alpha}=B_{n}\left(\alpha_{1}, \ldots, \alpha_{(N-1)}, \alpha\right), \alpha=\alpha_{N}, \\
& B_{n}\left(\alpha_{1}, \ldots, \alpha_{(N-1)}, \alpha\right)=\beta_{n}\left(\alpha_{1}, \ldots, \alpha_{(N-1)}, \alpha\right) / \beta_{N}\left(\alpha_{1}, \ldots, \alpha_{(N-1)}, \alpha\right), \\
& \alpha_{n}=\sum_{k \geq 1} f_{n k} \alpha^{k}, \quad n=1,2, \ldots, N-1 .
\end{aligned}
$$

Generally speaking, the integrals of motion may explicitly depend on time, $H_{n}=H_{n}(\alpha, t), 1 \leq n \leq N$. Introducing extra coupling constant $\alpha_{N+1}=t, \beta_{N+1}=1$, we may reduce $N$-dimensional case with time dependent integrals, to the $N+1$-dimensional case of time independent integrals. The motion equations can be derived from the integrals of motion:

$$
0=\dot{H}_{n}=\partial_{t} H_{n}+\frac{\partial H_{n}}{\partial \alpha_{m}} \dot{\alpha}_{m} \Rightarrow \dot{\alpha}_{n}=\beta_{n}(\alpha)=\frac{\operatorname{det} A_{n}}{\operatorname{det} A}, A_{n m}^{-1}=\frac{\partial H_{n}}{\partial \alpha_{m}} .
$$

When the number of time dependent integrals are $M<N$, we may derive for $M$ coupling constants RD equations and the remaining $N-M$ coupling constants maybe considered as control parameters. Defining some $N-M$ criteria of optimality, we may find corresponding optimal trajectories.

Solitons are particlelike states, solutions of motion equations and they quantum extensions. Examples are solitons of the sine-Gordon motion equation or barions-skirmions of the Skyrme model [16]. In particle theory, the skyrmion was described by Tony Skyrme in 1962 and consists of a quantum superposition of baryons and resonance states. Skyrmions as topological objects are important in solid state physics. Researchers could read and write skyrmions using scanning tunneling microscopy. The topological charge, representing the existence and non-existence of skyrmions, can represent the bit states "1" and "0".

QCD consists of quarks and gluons. Quarks possess both color $(r, g, b)$ and flavor $(u, d, s$, etc.), while gluons possess color $(r, g, b)$ and anti-color $(\bar{r}, \bar{g}, \bar{b})$, but not flavor. An open string (a string with two endpoints) is ideally suited to account for such quantum numbers at its two ends. For quarks, one end represents color and the other end - flavor. For gluons, one end represents color and the other - anti-color. In string theory, there are branes (higher dimensional extended objects that are generalized membranes) to which the endpoints of an open string are confined. Applying this idea to QCD, we introduce $N_{c}$ colored branes and $N_{f}$ flavored branes at which open strings corresponding to quarks and gluons terminate. The energy of a string is given by the sum of the classical energy stored inside the string and the excitation energies of vibration and rotation. Because the classical energy of a string is proportional to its length and because gluons are massless, $N_{c}$ colored branes should lie on top of each other. On the other hand, quarks possess intrinsic masses, and therefore the endpoints of a quark string, namely, a flavored brane and a colored brane should be separated from each other by a nonvanishing distance $U$. Then, the intrinsic quark mass $m_{q}$ can be 
represented as $m_{q}=U \times($ string tension), where the string tension is the energy stored inside a unit length of string and is represented: string tension $=1 /\left(2 \pi \alpha^{\prime}\right)$ in terms of $\alpha^{\prime}$, historically called the Regge slope. To describe QCD, we have to prepare Dp-branes and Dq-branes with $\mathrm{p}, \mathrm{q} \geq 3$ for colored branes and flavored branes, respectively, and these branes should be located in the space of more than five dimensions. To evaluate the amplitude for a certain process to occur in the above picture, we have to sum up all the possible two-dimensional world sheets with the weight $\exp (i S)$, where the action $S$ is given by $S=($ energy $) \times($ time $)=($ area of the string's world sheet $) / 2 \pi \alpha^{\prime}$, following the Feynman path integral formulation.

Cumulative Effect: Production of particles from nuclei in a region, kinematically forbidden for reactions with free nucleons is connected to the existence of Fluctons - droplets of dense cold nuclear matter. The size of fluctons is the same as nucleon one. The density of fluctons is several times more than the nucleon one. So, it seems that there are violation of the baryon number conservation and Pauli principle inside fluctons. The Pauli principle can be saved (small violation remains) if we suppose that small nucleons inside fluctons have a small color charge as indicate numerical experiments [1]: $\alpha_{s}$ beyond the critical size (and value) decreases toward zero - IR freedom.

Classical fields have canonical, rational for integer $D$, (mass-)dimensions. E.g. in electrodynamics

$$
\begin{aligned}
& L=\int d^{D} x\left(\bar{\psi}(\gamma(\partial-e A)-m) \psi-\frac{1}{4} F^{2}\right), \\
& d_{\psi}=[\psi]=(D-1) / 2, d_{A}=(D-2) / 2, d_{e}=(4-D) / 2 .
\end{aligned}
$$

Quantum corrections introduce (anomaly) corrections to the canonical dimensions, so the fields and coupling constants become fractals [11]. At fixed points of RD, the fractals are self similar and their compositions present at low energy unparticles, [7]. We will illustrate the qualitative picture of the (un)particle(like) objects with the simplest model of a scalar field given by the following Lagrangian

$$
L=L(\Phi, M, \lambda)=\frac{1}{2}\left(\partial_{\mu} \Phi\right)-\frac{1}{2} M^{2} \Phi^{2}-V(\Phi), \mu=0,1,2, \ldots, D
$$

where we usually take the self interaction in the form

$$
V(\Phi)=\lambda \Phi^{n}, n=-2,1,2,3,4,6 .
$$

In renormalizable case,

$$
n=\frac{2 D}{D-2}=2+\varepsilon(D), \varepsilon(D)=\frac{4}{D-2}, D=\frac{2 n}{n-2}=2+\varepsilon(n), \varepsilon(n)=\frac{4}{n-2},
$$

sometimes we consider also intermediate values of $n$ and $D$ and other forms of $V$.

In the free (self non interacting) field (particle) approximation: $\lambda=0$, but in external gravitational field we have

$$
L=\sqrt{-g} L(\Phi, M, 0), g=\operatorname{detg}_{\mu \nu}(x) .
$$

Now we will see a nice composite particle mechanism :) Let us take a substitute: $\Phi=\varphi^{k}$, than we find

$$
L(g, \Phi, M)=L\left(\left(k \varphi^{k-1}\right)^{4} g, \varphi, M / k\right), g_{\mu v}(x) \Rightarrow\left(k \varphi^{k-1}\right)^{4 / D} g_{\mu v}(x) .
$$

Indeed

$$
\left.L(g, \Phi, M)=\sqrt{-g}\left(k^{2} \varphi^{2(k-1)} \frac{1}{2}\left(\partial_{\mu} \varphi\right)^{2}-\frac{1}{2} M^{2} \varphi^{2 k}\right)=\sqrt{-g\left(k \varphi^{k-1}\right)^{4}}\left(\frac{1}{2}\left(\partial_{\mu} \varphi\right)^{2}\right)-\frac{1}{2}\left(\frac{M}{k}\right)^{2} \varphi^{2}\right) .
$$


Now, having an experience with constituent - composite particle relation, we turn attention on the self-interaction term,

$$
L=\sqrt{-g\left(k \varphi^{k-1}\right)^{4}}\left(\ldots-\frac{\lambda}{k^{2}} \varphi^{N}\right), N=k n-2(k-1) .
$$

Most natural value of $n$ for stable systems $(1+1 \rightarrow 1+1,2 \rightarrow 2)$ is $n=4$. In this case, $N=2 k+2$ and only natural value of constituents for which we have a renormalizable interaction is $k=2 \Rightarrow N=6$ with corresponding space-time dimension $D=3$. The most natural value for fission-fusion interaction $(1 \leftrightarrow 2)$ is $n=3 \Rightarrow N=k+2$, for which we have realistic values $k=2$ and $N=4, D=4$ :) Other interesting values of naturally interpretable monomial (polynomial) interactions generally corresponds to the non-integer, fractional-fractal dimensions of space(time) $D$, with fractal-fluctonunparticle interpretations of the corresponding states of matter.

The size of particle-like states (solutions of the motion equations) is defined as $l \sim M^{-1}$, because at the boundary region, the linear part of the motion equations dominates and the Yukawa-like asymptotic $\Phi(r) \sim e^{-M r}$ acts. In a pion-nucleon model for nucleon size we have $l_{N} \sim m_{\pi}^{-1} \simeq 1.43 \mathrm{fm}$. The amplitude of the state (at maximum) $A \sim \lambda^{-\alpha}, \alpha=1 /(n-2)$. Indeed, the motion equation does not contain the coupling constant after a scaling substitution $\Phi=\lambda^{-\alpha} \phi$, so a particle-like solution $\phi$ does not contain $\lambda$ and corresponding solution $\Phi=\lambda^{-\alpha} \phi \sim \lambda^{-\alpha}$,

$$
\triangle \Phi+M^{2} \Phi+\lambda n \Phi^{n-1}=\lambda^{-\alpha}\left(\triangle \phi+M^{2} \phi+\lambda^{1-(n-2) \alpha} n \phi^{n-1}\right)=0 .
$$

At not so low energies from string theory we may extract the following scalar field theory

$$
L=\sqrt{-g}\left(\frac{1}{2}\left(\partial_{\mu} \Phi\right)^{2}-\frac{1}{2} M^{2} \Phi^{2}-\lambda \Phi^{3}\right), \mu=0,1, \ldots, D-1, D=6+\varepsilon,
$$

where $\varepsilon \in[0,20]$. The one-loop $\beta$-function is $\beta(a)=(D-6) a-\beta_{2} a^{2}, a \sim \lambda^{2}$ and it has stable UV fixed point at $a=(D-6) / \beta_{2}$ and IR fixed point $a=0$. Beyond this point we have an unparticle $\Phi=\phi^{2}$ with Lagrangian

$$
L=\sqrt{-g^{\prime}}\left(\frac{1}{2}\left(\partial_{\mu} \phi\right)^{2}-\frac{1}{2}\left(\frac{M}{2}\right)^{2} \phi^{2}-\frac{\lambda}{4} \phi^{4}\right), \mu=0,1, \ldots, d-1, d=4-\varepsilon, \varepsilon \in[0,1] .
$$

The one-loop $\beta$-function is $\beta(\lambda)=(d-4) \lambda+b \lambda^{2}$ and it has stable IR fixed point at $\lambda=(4-d) / b$. The UV fixed point is $\lambda=0$. At this point we have reduction from higher dimensional $\Phi^{3}$ to lower dimensional $\phi^{4}$. Another possibilities is an unparticle $\Phi=\varphi^{4}$ with Lagrangian

$$
L=\sqrt{-g^{\prime \prime}}\left(\frac{1}{2}\left(\partial_{\mu} \varphi\right)^{2}-\frac{1}{2}\left(\frac{M}{4}\right)^{2} \varphi^{2}-\frac{\lambda}{6} \varphi^{6}\right), \mu=0,1, \ldots, d-1 .
$$

The one loop $\beta$-function is $\beta(\lambda)=(d-3) \lambda+c \lambda^{2}, d=3-\varepsilon, \varepsilon \in[0,2]$. The IR fixed point is $\lambda=\varepsilon / c$. UV fixed point is $\lambda=0$. Similar consideration gives reduction from higher energy $\phi^{4}$ model to lower energy $\varphi^{6}$ one. Some technical questions remain. One of them concern to the substitution $\Phi=\phi^{2}$. It restricts $\Phi$ as $\Phi \geq 0$. Formulation of positivity condition is not so easy. We will take another path, we define the interaction as $\Phi^{3}=\left(\Phi^{2}\right)^{3 / 2} \geq 0$. Then the substitution $\Phi^{2}=\phi^{4}$ will work. By the way, by this definition we made also another improvement: the potential becomes bounded from below. For the reduction the substitution $\Phi^{2}=\phi^{4}$ also works,

$$
L=\sqrt{-g}\left(\frac{1}{8 \Phi^{2}}\right)\left(\partial_{\mu} \Phi^{2}\right)^{2}-\frac{1}{2} M^{2} \Phi^{2}-\lambda\left(\Phi^{2}\right)^{n / 2}, n=3,4
$$

Note that by substitution

$$
\left(\frac{\Phi}{\Phi_{0}}\right)^{2}=\phi^{2 k}, \phi^{2}=\exp \left(\ln \left(\Phi^{2} / \Phi_{0}^{2}\right) / k\right)=1+\frac{1}{k} \ln \left(\frac{\Phi}{\Phi_{0}}\right)^{2}+O\left(K^{-2}\right), \phi= \pm 1+O\left(k^{-1}\right)
$$


we reduce the field theory to a discrete theory, to a system of bits. Also, changing dimension of space $D$ and nonlinearity $n$ restricted by condition

$$
n=\frac{2 D}{D-2}, D=\frac{2 n}{n-2}, \frac{1}{n}+\frac{1}{D}=\frac{1}{2}
$$

we assume that they are functions of scale or coupling constant, due to monotonic property of the coupling constant. We have the following relation

$$
\beta_{n}=-\frac{4}{(D-2)^{2}} \beta_{D}, \beta_{n}=\mu \frac{d n}{d \mu}=\frac{d n}{d \lambda} \beta_{\lambda}, \beta_{D}=\mu \frac{d D}{d \mu}=\frac{d D}{d \lambda} \beta_{\lambda}
$$

\section{References}

[1] I.L. Bogolubsky, E.M. Ilgenfritz, M. Müller-Preussker, A. Sternbeck, Phys. Lett. B 676, 69 (2009)

[2] S.J. Brodsky, G.F. de Tèramond, A. Deur, Phys. Rev. D 81, 096010 (2010)

[3] J.C. Collins, Renormalization (Cambrige Univ. Press, London, 1984)

[4] D. Diakonov, Prog. Par. Nucl. Phys. 51, 173 (2003)

[5] M. Duff, L. Okun, G. Veneziano, J. High Energy Phys. 0203, 023 (2002)

[6] L.D. Faddeev and L.A. Takhtajan, Hamiltonian methods in the theory of solitons (Springer, Berlin, 1990)

[7] H. Georgi, Phys. Rev. Lett. 98, 221601 (2007)

[8] S.V. Ketov, Quantum Non-linear Sigma-models (Springer, Berlin, 2000)

[9] N. Koblitz, p-adic numbers, p-adic analysis, and Zeta-functions (Springer, Berlin, 1977)

[10] J.M. Maldacena, Adv. Theor. Math. Phys. 2, 231 (1988)

[11] N. Makhaldiani, Physics of Particles and Nuclei Letters 8, 325 (2011)

[12] N. Makhaldiani, Atomic Nuclei 74, 1040 (2011)

[13] N. Makhaldiani, Physics of Particles and Nuclei Letters 11, 1019 (2014)

[14] L. Okun, Sov. Phys. Usp. 34, 818 (1991)

[15] M. Planck, Ann. d. Phys. 1, 69 (1900)

[16] T. Skyrme, Nuclear Physics 31, 556 (1962)

[17] G. Stoney, Phil.Mag. 11, 381-391 (1881)

[18] M.B. Voloshin, K.A. Ter-Martyrosian, Gauge Theory of Elementary Particles (Atomizdat, Moscow, 1984) 